\title{
Array Processing and Fast Optimization Algorithms for Distorted Circular Contour Retrieval
}

\author{
Julien Marot and Salah Bourennane \\ GSM, Institut Fresnel, CNRS-UMR 6133, Ecole Centrale Marseille, Université Aix-Marseille III, D.U. de Saint Jérôme, \\ 13397 Marseille Cedex 20, France
}

Received 19 July 2006; Revised 20 December 2006; Accepted 17 February 2007

Recommended by Wilfried Philips

A specific formalism for virtual signal generation permits to transpose an image processing problem to an array processing problem. The existing method for straight-line characterization relies on the estimation of orientations and offsets of expected lines. This estimation is performed thanks to a subspace-based algorithm called subspace-based line detection (SLIDE). In this paper, we propose to retrieve circular and nearly circular contours in images. We estimate the radius of circles and we extend the estimation of circles to the retrieval of circular-like distorted contours. For this purpose we develop a new model for virtual signal generation; we simulate a circular antenna, so that a high-resolution method can be employed for radius estimation. An optimization method permits to extend circle fitting to the segmentation of objects which have any shape. We evaluate the performances of the proposed methods, on hand-made and real-world images, and we compare them with generalized Hough transform (GHT) and gradient vector flow $(\mathrm{GVF})$.

Copyright (c) 2007 J. Marot and S. Bourennane. This is an open access article distributed under the Creative Commons Attribution License, which permits unrestricted use, distribution, and reproduction in any medium, provided the original work is properly cited.

\section{INTRODUCTION}

Circular features in digital images are sought very often in digital image processing. An image containing one or several contours is composed of black pixels with value " 1 " which represent the contours, over a white background with pixel value " 0 ." Circle fitting, in particular, is faced in several application fields such as quality inspection for food industry and mechanical parts, fitting particle trajectories $[1,2]$. Circle fitting also has applications in microwave engineering, and ball detection in robotic vision systems [3]. Several methods have been proposed for solving this problem using, among others, the generalized Hough transform (GHT) [4, 5], array processing methods $[6,7]$, contour-based snakes methods $[8,9]$. The formalism proposed by Aghajan [6] permits to detect circular or elliptic contours. The coordinates of the center of a circle are estimated by an array processing method [6] that works on virtual signals generated from the image. Each row or column of the image is associated with a sensor of a linear antenna.

In this paper, we propose a new approach which employs a circular antenna for the estimation of the radius of a circle, and we propose to adapt an optimization method to retrieve the distortions between any nearly-circular contour and a circle. We adopted a similar strategy in [10], in the case of the retrieval of approximately rectilinear distorted contours, by means of a uniform linear antenna.

We choose to employ either the fixed step gradient method or DIRECT [11] combined with spline interpolation as an optimization method for the retrieval of the distortions between the expected distorted contour and a circle that is a rough approximation of this contour.

The rest of the paper is organized as follows: in Section 2, we set the problem of circle retrieval and show how to model a circular antenna. In Section 3, we explain why signal generation out of an image containing circles permits to obtain linear phase signals when the proposed circular antenna is used. By using a Minimum Description Length (MDL) criterion, we retrieve the number of concentric circles; then with a high-resolution method, we estimate the radius of the expected circles. In Section 4, we derive the numerical complexity of our method and compare it with the complexity of GHT. In Section 5, we propose to extend the work concerning circular contours to any circular-like contour. In order to adapt the optimization methods proposed in $[10,12]$, we simulate the generation of signals from the image on a 
circular antenna with a constant propagation parameter. In Section 6 we present the results obtained by all proposed methods through an application to hand-made and realworld images. We compare the performances of the proposed methods to those of GHT [5] and GVF [8].

\section{PROBLEM SETTING AND VIRTUAL SIGNAL GENERATION}

Our purpose is to estimate the radius of a circle, and the distortions between a closed contour and a circle that fits this contour. We propose to employ a circular antenna that permits a particular signal generation.

\subsection{Problem setting}

Figure 1(a) presents a binary digital image $I$. An object in the image is made of edge pixels with value " 1 ," over a background of zero-valued pixels. The object is close to a circle with radius value $r$ and center coordinates $\left(l_{c}, m_{c}\right)$. Figure 1(b) shows a subimage extracted from the original image, such that its top left corner is the center of the circle. We associate this subimage with a set of polar coordinates $(\rho, \theta)$, such that each pixel of the expected contour in the subimage is characterized by the coordinates $(r+\Delta \rho, \theta)$, where $\Delta \rho$ is the shift between the pixel of the contour and the pixel of the circle that roughly approximates the contour and which has the same coordinate $\theta$. We seek for star-shaped contours, that is, contours that can be described by the relation $\rho=f(\theta)$, where $f$ is any function that maps $[0,2 \pi]$ to $\mathbb{R}_{+}$. The point with coordinate $\rho=0$ corresponds then to the center of gravity of the contour. For instance to the center in the case of a circle. A classical method of finding the parameters of circles is the generalized Hough transform (GHT) [4]. More details about a fast version of GHT are available in [5]. We apply

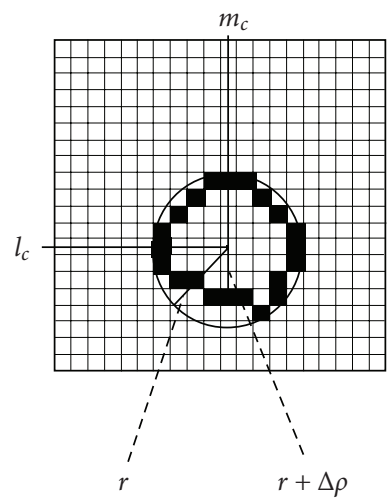

(a)

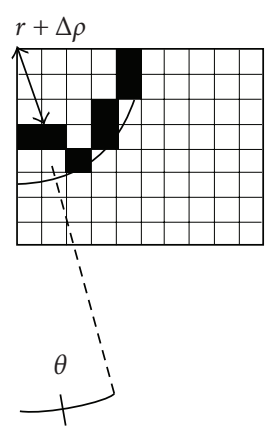

(b)
Figure 1: (a) An image containing a contour close to a circle with center coordinates $\left(l_{c}, m_{c}\right)$; (b) bottom right quarter of the contour and pixel coordinates in the polar system $(\rho, \theta)$ having its origin on the center of the circle. $r$ is the radius of the circle. $\Delta \rho$ is the value of the shift between a pixel of the contour and the pixel of the circle having the same coordinate $\theta . \Delta \rho$ can be either positive or negative. the GHT to obtain the radius of concentric circles when their center is known. Its basic principle is to count the number of pixels that are located on a circle for all possible radius values. The estimated radius value corresponds to the maximum number of pixels. Some faster versions were proposed [5], which avoid the application of the Laplacian operator on the whole image and restrict the possible radius values to an a priori-fixed interval. However, the drawback of GHT is still its elevated computational load.

Hence, there is a need for a faster procedure for estimating the radius. In [7], Aghajan and Kailath proposed to replace the Hough transform by the SLIDE algorithm for retrieving straight lines. SLIDE relies on faster algorithms, the so-called high-resolution methods of array processing [7]. Therefore, we expect that such methods lead to faster algorithms for circle detection as well, compared to GHT.

The existing methods that combine array processing with optimization methods employ a signal generation scheme such that only one unknown parameter of the optimization problem is contained in one component of the generated signal. In previous work $[10,12]$, the optimization method that is set retrieves the phase shift between a linear phase model and the phase of a signal which is generated from the image. The phase shift corresponding to each component of the signal generated on a linear antenna is proportional to the pixel shift between an approximately-linear contour made of one pixel per row or column and an initialization straight contour. The purpose of this paper is to retrieve contours which are no longer approximately linear but approximately circular. Contours which are approximately circular are supposed to be made of more than one pixel per row for some of the rows and more than one pixel per column for some columns. Therefore, the principles of signal generation which are relevant for the retrieval of approximately linear contours are no longer relevant for nearly circular contours.

Section 2.2 shows how to associate one sensor of the antenna with one specific orientation in the image for signal generation.

\subsection{Virtual signal generation}

We set an analogy between the estimation of a circular contour in an image and the estimation of a wavefront in array processing. Our basic idea is to obtain a linear phase signal from an image containing a contour which is a quarter of circle. The phase of the signals which are virtually generated on the antenna is constant or varies linearly as a function of the index of the sensor.

A quarter of circle with radius $r$ and a circular antenna are represented on Figure 2. We explain here how to generate signal components along several lines in the image, corresponding to different values of $\theta$ in the polar coordinate system of the subimage. The antenna is associated with the subimage containing any quarter of the expected contour. It is a quarter of circle centered on the top left corner, and going through the bottom right corner of the subimage. Such an antenna is adapted to the subimages containing each quarter of the expected contour (see Figure 2). In practice, 


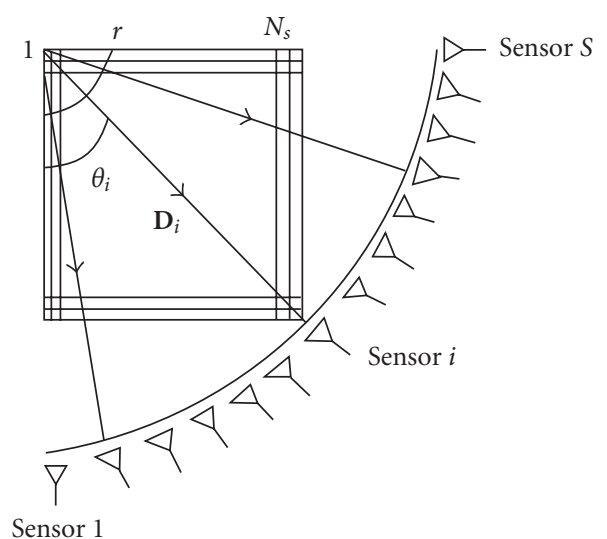

FIgURE 2: A subimage is extracted from the processed image: its top left corner is the center of the expected circle of radius $r$. The subimage is associated with a circular array composed of $S$ sensors.

the extracted subimage is possibly rotated such that its top left corner is the estimated center. A squared image is obtained by zero-padding. Therefore, the antenna has radius $R_{\text {antenna }}$ such that $R_{\text {antenna }}=\sqrt{2} \cdot N_{\text {subimage }}$, where $N_{\text {subimage }}$ is the number of rows or columns in the subimage. When we consider the subimage which includes the right bottom part of the expected contour, we have the relation $N_{\text {subimage }}=$ $\max \left(N-l_{c}, N-m_{c}\right)$, where $l_{c}$ and $m_{c}$ are the vertical and horizontal coordinates of the center of the expected contour in a Cartesian set centered on the top left corner of the whole processed image (see Figure 1). Coordinates $l_{c}$ and $m_{c}$ are estimated by the method proposed in [6], which is based on the generation of signals on a linear antenna by a variable speed propagation scheme.

The signal generation scheme on a circular antenna is such that the directions adopted for signal generation go from the top left corner of the subimage to the corresponding sensor. If the antenna is composed of $S$ sensors, there are $S$ signal components. Let us consider $\mathbf{D}_{\mathbf{i}}$, the line that makes an angle $\theta_{i}$ with the vertical axis and goes through the top left corner of the subimage. The $i$ th component $z(i)(i=1, \ldots, S)$ of the signal $\mathbf{z}$ generated out of the image is given by

$$
z(i)=\sum \sum_{\substack{l, m=1 \\(l, m) \in \mathbf{D}_{\mathbf{i}}}}^{l, m=N_{\text {subimage }}} I(l, m) \exp \left(-j \mu \sqrt{l^{2}+m^{2}}\right) .
$$

The integer $l$ (resp., $m$ ) indexes the lines (resp., the columns) of the image. Parameter $\mu$ is the propagation parameter [13]. Each sensor indexed by $i$ is associated with a line $\mathbf{D}_{\mathbf{i}}$ having an orientation $\theta_{i}=((i-1) \cdot \pi / 2) / S$. The constraint $(l, m) \in \mathbf{D}_{\mathbf{i}}$, that is, the pixel with coordinates $(l, m)$ belongs to the line with orientation $\theta_{i}$, is performed in two steps: let set $l$ be the set of indexes along the vertical axis, set $m$ the set of indexes along the horizontal axis, if $\theta_{i}$ is less than or equal to $\pi / 4$, set $l=\left[1: N_{\text {subimage }}\right]$ and set $m=\lfloor[1$ : $\left.\left.N_{\text {subimage }}\right] \cdot \tan \left(\theta_{i}\right)\right]$, if $\theta_{i}$ is greater than $\pi / 4$, set $m=[1$ : $\left.N_{\text {subimage }}\right]$ and set $l=\left\lfloor\left[1: N_{\text {subimage }}\right] \cdot \tan \left(\pi / 2-\theta_{i}\right)\right\rfloor$. Symbol $\lfloor\cdot\rfloor$ means integer part. Dividing the generation process in two steps allows to work with low-valued angles and obtain lower errors when integer parts are computed. The minimum number of sensors that permits a perfect characterization of any possibly distorted contour is the number of pixels that would be virtually aligned on a quarter of circle having radius $\sqrt{2} \cdot N_{\text {subimage. }}$ Therefore, the minimum number $S$ of sensors is $\sqrt{2} \cdot N_{\text {subimage. }}$ In this equation, the presence of the term $(l, m) \in \mathbf{D}_{\mathbf{i}}$ shows that only the pixels of the image that are crossed by line $\mathbf{D}_{\mathbf{i}}$ are taken into account for signal generation. The term $I(l, m)$ indicates that only pixels that have value different from 0 are taken into account for signal generation.

\section{PROPOSED METHOD FOR RADIUS ESTIMATION}

In the most general case, there exists more than one circle for one center. We show how several possibly-close radius values can be estimated with a high-resolution method. For this, we use a variable speed propagation scheme toward circular antenna. We propose a method for the estimation of the number $d$ of concentric circles, and the determination of each radius value. For this purpose, we employ a variable speed propagation scheme [13]. We set $\mu=\alpha(i-1)$, for each sensor indexed by $i=1, \ldots, S$. From (1), the signal received on each sensor is

$$
z(i)=\sum_{k=1}^{d} \exp \left(-j \alpha(i-1) r_{k}\right)+n(i), \quad i=1, \ldots, S,
$$

where $r_{k}, k=1, \ldots, d$ are the values of the radius of each circle, and $n(i)$ is a noise term that can appear because of the presence of outliers. All components $z(i)$ compose the observation vector $\mathbf{z}$. From the observation vector we build $K$ vectors of length $M$ with $d<M \leq S-d+1$. Note that the number of sensors can be chosen relatively low, as soon as $S>d$ : the linear phase relationship holds whatever the number of sensors $S$. In order to maximize the number of subvectors [10], we choose $K=S+1-M$. By grouping all subvectors in matrix form, we obtain

$$
\mathbf{Z}_{K}=\left[\mathbf{z}_{1}, \ldots, \mathbf{z}_{K}\right],
$$

where

$$
\mathbf{z}_{l}=\mathbf{A}_{M} \mathbf{s}_{l}+\mathbf{n}_{l}, \quad l=1, \ldots, K .
$$

$\mathbf{A}_{M}=\left[\mathbf{a}\left(r_{1}\right), \ldots, \mathbf{a}\left(r_{d}\right)\right]$ is a Vandermonde type matrix of size $M \times d$,

$$
\begin{aligned}
\mathbf{a}\left(r_{k}\right)=[ & {\left[1, \exp \left(-j \alpha r_{k}\right), \exp \left(-j \alpha 2 r_{k}\right), \ldots,\right.} \\
& \left.\exp \left(-j \alpha(S-1) r_{k}\right)\right]^{T}
\end{aligned}
$$

$T$ denotes transpose, $\mathbf{s}_{l}=[1,1, \ldots, 1]^{T}$ is a length $d$ vector with all values equal to one.

The signal model of (4) suits the frequency estimation method Estimation of Parameters by Rotational Invariance Techniques (ESPRIT) proposed in [14] and TLS-ESPRIT, a Total Least Squares version of ESPRIT. We choose to employ the subspace-based method TLS-ESPRIT, which has exhibited a good behavior in the application of array processing to straight line detection [15]. TLS-ESPRIT works on 
the measurements obtained from two overlapping subarrays, and falls into two parts: the estimation of a covariance matrix and the application of a total least squares criterion. The estimated radius values are obtained in the same way as the orientation of straight lines are obtained in [13]:

$$
\hat{r}_{k}=\frac{1}{\alpha} \ln \left(\ln \left(\frac{\lambda_{k}}{\left|\lambda_{k}\right|}\right)\right), \quad k=1, \ldots, d,
$$

where $\ell m$ denotes imaginary part, $\left\{\lambda_{k}, k=1, \ldots, d\right\}$ are the eigenvalues of a diagonal unitary matrix that relates the measurements from the first subarray to the measurements resulting from the second subarray. At this point, any circle is characterized by its center coordinates and its radius.

\section{NUMERICAL COMPLEXITY OF THE METHODS}

In the general case, the image contains outlier pixels and several concentric circles. First, as concerns the estimation of the coordinates of the center [6]: it is performed by signal generation upon a linear antenna located on one horizontal and then one vertical side of the image, followed by TLS-ESPRIT method. This antenna contains $N$-sensors, each corresponding to one row or column. The estimation of the coordinate of the center requires the following operations and computational complexity, for each coordinate along horizontal and vertical axes [6]:

(i) variable speed propagation scheme upon a linear antenna aside the image: $N^{2}$ operations [7];

(ii) application of TLS-ESPRIT to the covariance matrix of the generated signals: for the estimation, and respectively the fast eigendecomposition, of the covariance matrix in TLS-ESPRIT method $[13,16]: N \cdot M$, and, respectively, $M^{2}$.

We choose $M=\sqrt{N}$, as recommended in [13]. The computational complexity for center retrieval is then $N^{2}+N$. $(\sqrt{N}+1)$.

As concerns the estimation of the radius values, we remind that $d$ is the number of concentric circles and the dimension of the signal subspace in the covariance matrix in TLS-ESPRIT method. The computational complexity of the steps of our method for radius estimation is

(i) for signal generation $[7,16]$ : the number of sensors multiplied by the number of pixels that are crossed by each line $\mathbf{D}_{\mathbf{i}}$, that is, $S \cdot N_{\text {subimage }}$ or equivalently $S \cdot N$;

(ii) for the estimation, and, respectively, the fast eigendecomposition, of the covariance matrix in TLS-ESPRIT method [13, 16]: $S \cdot M$, and, respectively, $d \cdot M^{2}$.

We choose $M=\sqrt{S}$, as recommended in [13]. The computational complexity of the angle estimation method is then $S \cdot N+S \cdot(\sqrt{S}+d)$. In practice, the order of magnitude of $S$ is $N$, and the computational load of the proposed method for center and radius estimation is $N^{2}$.

As concerns the generalized Hough transform, we discretize the $\rho$ axis to the minimum required number of values, that is, $\sqrt{2} \cdot N$ for the computation of the accumulator. Also, the $\theta$ axis for counting the edge pixels is discretized to $\sqrt{2} \cdot N$ values $(\sqrt{2} \cdot N$ is the minimum number of orientations that permits to characterize any contour in the image, see Section 2.2). In these conditions, the order of magnitude of the computational load of the generalized Hough transform, for the estimation of the center and the radius of the circles, is $N^{3}$ [5]. To conclude, the computational complexity of the proposed method is $N^{2}$, as compared to $N^{3}$ for the generalized Hough transform. The same order of magnitude of computational loads was obtained in [7] when SLIDE algorithm was compared with the Hough transform for straight line retrieval.

\section{OPTIMIZATION METHOD FOR THE ESTIMATION OF NEARLY CIRCULAR CONTOURS}

The optimization methods proposed in $[10,12]$ assume that one component of the generated signal is associated with only one unknown for the optimization method, namely the pixel shift between the initialization contour and the expected contour at one row (or column) of the image. We propose to employ a circular antenna and to retrieve the shift values between an initialization circle and the expected contour, along several directions in the image. These directions go through the center of the initialization circle and have several orientations.

We work successively on each quarter of circle, and retrieve the distortions between one quarter of the initialization circle and the part of the expected contour that is located in the same quarter of the image. As an example, in Figure 1, the right bottom quarter of the considered image is represented in Figure 1(b). Here is an optimization strategy inspired by [10]: a contour in the considered subimage can be described in a set of polar coordinates by $\{\rho(i), \theta(i), i=$ $1, \ldots, S\}$. We aim at estimating the $S$ unknowns $\rho(i)$, $i=1, \ldots, S$ that characterize the contour, forming a vector

$$
\boldsymbol{\rho}=[\rho(1), \rho(2), \ldots, \rho(S)]^{T} .
$$

The basic idea is to consider that $\rho$ can be expressed as $\boldsymbol{\rho}=[r+\Delta \rho(1), r+\Delta \rho(2), \ldots, r+\Delta \rho(S)]^{T}$ (see Figure 1), where $r$ is the radius of a circle that approximates the expected contour. The optimization method that we employ aims at estimating $\{\Delta \rho(i), i=1, \ldots, S\}$, that is, the shifts between the initialization circle and the expected contour.

By making an analogy with (2) and keeping a constant propagation parameter, the components of signal $\mathbf{z}$ generated out of the image containing the expected contour are the following:

$$
z(i)=\exp (-j \mu \rho(i)), \quad \forall i=1, \ldots, S .
$$

Equation (8) is obtained from (2) by replacing one constant $r_{k}$ by a radial coordinate $\rho(i)$, that can be different for each sensor $i$. So we try to recreate the signal defined in (8) from which we ignore the $S$ parameters. We start from an initialization vector $\rho_{0}$, characterizing a quarter of circle that approximates the expected distorted contour in the considered subimage. The $S$ components of $\boldsymbol{\rho}_{0}$ are equal to $\hat{r}$, the radius value that was previously estimated $\rho_{0}=[r, r, \ldots, r]^{T}$. Then, 
with $k$ indexing the steps of this recursive algorithm, we minimize

$$
J\left(\boldsymbol{\rho}_{k}\right)=\left\|\mathbf{z}-\mathbf{z}_{\text {estimated for } \boldsymbol{\rho}_{k}}\right\|^{2},
$$

where $\|\cdot\|$ represents the norm induced by the usual scalar

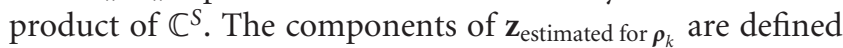
in the same way as the components of $\mathbf{z}$ as a function of the components of $\boldsymbol{\rho}_{k}$, and the components of $\boldsymbol{\rho}_{k}$ are obtained from the components of $\boldsymbol{\rho}_{0}$ by adding a shift $\boldsymbol{\rho}_{k}=$ $\left[r+\Delta \rho_{k}(1), r+\Delta \rho_{k}(2), \ldots, r+\Delta \rho_{k}(S)\right]^{T}$. In this paper, we use the fixed step gradient method. The variable step gradient method could also be used. The vectors of the series are obtained by the relation

$$
\forall k \in \mathbb{N}: \boldsymbol{\rho}_{k+1}=\boldsymbol{\rho}_{k}-\lambda \nabla\left(J\left(\boldsymbol{\rho}_{k}\right)\right),
$$

where $0<\lambda<1$ is the step for the descent. The recurrence loop is

$$
\boldsymbol{\rho}_{k} \longrightarrow \mathbf{z}_{\text {estimated for } \boldsymbol{\rho}_{k}} \longrightarrow J\left(\boldsymbol{\rho}_{k}\right) .
$$

The gradient is estimated using finite differences. When $k$ tends to infinity, the criterion $J$ tends to zero and $\rho_{k}(i)=$ $r+\Delta \rho(i)=\rho(i)$, for all $i=1, \ldots, S$.

We denote by $\hat{\boldsymbol{\rho}}$ the vector containing all estimated values $\rho_{k}(i), i=1, \ldots, S$, with $k$ tending to infinity. A more elaborated method based on DIRECT algorithm and spline interpolation can be adopted in order to reach the global minimum of the criterion $J$ of (9) to be minimized. This

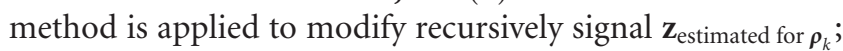
at each step of the recursive procedure vector $\boldsymbol{\rho}_{k}$ is computed by making an interpolation between some "node" values that are retrieved by DIRECT.

The interest of the combination of DIRECT with spline interpolation comes from the elevated computational load of DIRECT. Details about DIRECT algorithm are available in [11]. Its main property is that it is a global optimization method it permits to obtain the global minimum of a function. DIRECT normalizes the research space in a hypercube and evaluates the solution which is located in the center of this hypercube. Then, some solutions are evaluated and the hypercube is divided into smaller cubes supporting the zones were the evaluations are small. Let $O$ be an integer lower than $S$. A cubic spline $f$ interpolating on the partition $y(1), \ldots, y(O)$ that we call "node points," to the elements $\rho(1), \ldots, \rho(S)$, is a function for which $f(y(k))=\rho(k)$ for $k=1, \ldots, O$. It is a piecewise polynomial function that consists of $O-1$ cubic polynomials $f_{k}$ defined on the ranges $[y(k), y(k+1)]$. Furthermore, each $f_{k}$ is joined at $y(k)$, for $k=2, \ldots, O-1$, such that $\rho^{\prime}(k)=f^{\prime}(y(k))$ and $\rho^{\prime \prime}(k)=$ $f^{\prime \prime}(y(k))$ are continuous. The $k$ th polynomial curve, $f_{k}$, is defined over the fixed interval $[y(k), y(k+1)]$ and is a thirdorder polynomial. Then interpolation provides an approximate value of $S$ elements starting from $O<S$ elements.

Spline interpolation permits to obtain a continuous estimated contour and cubic splines provide a good compromise between computational load and accuracy of the interpolation.
The computational load of DIRECT algorithm grows rapidly when the number of sensors, or equivalently the number of unknown phase values, increases. We accelerate DIRECT algorithm by reducing the number of retrieved unknowns and then we propose spline interpolation to obtain the $S$ components of $\hat{\rho}$; we interpolate a subset of values of $\boldsymbol{\rho}_{k}$, which are retrieved by DIRECT algorithm. The more the interpolation nodes are, the more precise the estimation becomes, but the slower the algorithm becomes.

\section{RESULTS OBTAINED BY THE PROPOSED METHODS}

We apply here the proposed methods to hand-made and realworld images. First we compare our methods based on signal generation upon a circular antenna with the generalized Hough transform (GHT). Secondly we compare our methods with gradient vector flow (GVF) when images with distorted contours are considered. The efficiency of the proposed methods is measured from the final result thanks to the criterion $M E_{\rho}$, which is the mean error over the estimation of coordinates of the pixels of the curve. For the four quarters of an image, the coordinates of the pixels of the curve are contained in vector $\rho$ defined in (7), and their estimates are contained in vector $\hat{\boldsymbol{\rho}} . M E_{\boldsymbol{\rho}}$ is defined by

$$
M E_{\boldsymbol{\rho}}=\frac{1}{S} \sum_{i=1}^{S}|\hat{\boldsymbol{\rho}}(i)-\boldsymbol{\rho}(i)|,
$$

where $|\cdot|$ stands for absolute value. The error over all pixels of the contour is the mean of the error obtained with each quarter of image. When several contours are retrieved for one image, the mean value of the error over all contours is provided.

\subsection{Circle retrieval}

The proposed method for circle fitting is applied to handmade and real-world images having $N=200$ columns and rows. We adopt a number of sensors $S=400$ for each quarter of image, which is larger than the minimum acceptable value. Procedures for center and radius estimation are run with propagation parameter $\alpha=1.35 \cdot 10^{-2}$. When TLSESPRIT method is run the length of each subarray, as recommended in [13], $M=\sqrt{S}=20$. The signal generation scheme dedicated to distortion estimation is run with constant propagation speed $\mu=5 \cdot 10^{-3}$. This value avoids phase indetermination [12].

\subsubsection{Nonnoisy image: computational times}

We first considered the case of an image containing two concentric circles. Thanks to the adopted formalism, this problem is equivalent to the resolving of two close-valued frequencies in array processing. High-resolution methods were specifically created to face this problem and exhibited a very good behavior [14]. In this case, starting from the signals generated on the circular antenna, MDL criterion permits to estimate the number of expected circles, and the highresolution method TLS-ESPRIT manages to estimate the 


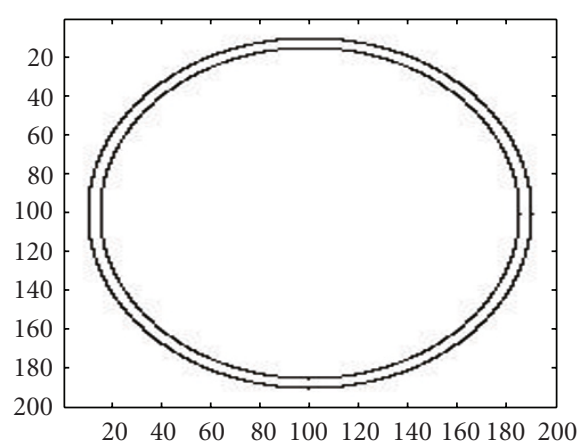

(a)

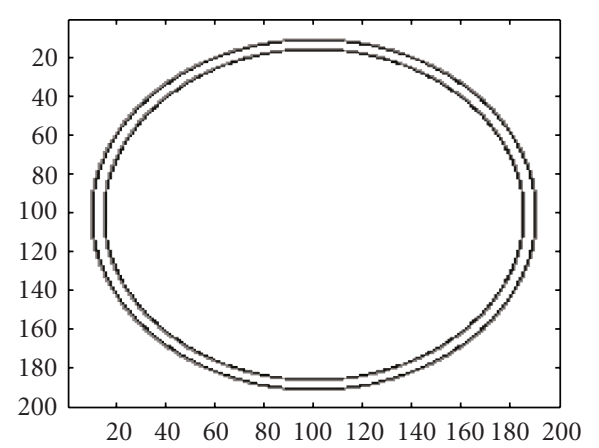

(b)

FIGURE 3: Center and radius estimation, two circles: (a) processed, (b) result (superimposed), with the proposed method for radius estimation or equivalently with GHT: $M E_{\rho}=0.1$ (resp., 0.3 for GHT).

radius of each circle. The expected radius values are 85 and 90 pixels (see Figure $3(\mathrm{a})$ ) thus, differing by only $6 \%$. The estimated radius values obtained with the proposed method are 85.1 and 89.9 pixels, and the required computational time is 0.359 seconds, on a $3.0 \mathrm{GHz}$ Pentium 4 PC running Windows. The same processor and the same software are used throughout all experiments. The slight bias may come from the signal generation process. When GHT is applied to obtain an estimation of the radius values, $\rho$ and $\theta$ parameters are both quantized to $S$ values to create the accumulator. Estimated radius values are 84.7 and 90.3 pixels, and the required computational time is 2.2 seconds. Visually, there is no difference between the results of both methods (see Figure 3(b)).

\subsubsection{Noisy images: statistical results}

We now consider the case of a noisy image. High-resolution methods are known to cope with noisy signals. In particular, TLS-ESPRIT method works optimally in the case of uncorrelated white noise [14]. This condition holds for signals generated out of an image by a propagation scheme, they are impaired by an uncorrelated white Gaussian noise if the noisy pixels are randomly distributed in the image [7]. This permits to predict that TLS-ESPRIT method should work optimally with this kind of noisy image. We performed a statistical study (1000 trials) in order to compare the robustness of the proposed method and the generalized Hough transform, for radius estimation. In order to impair our hand-made images, we add $20 \%$ of Gaussian noise with mean 0.02 and standard deviation 0.009 . Figure 4 shows an example of processed image containing a circle with radius value $r$, and the result obtained with the proposed method (see Figure 4(a)), and an example of processed image and the result obtained with GHT (see Figure 4(b)). Mean error $M E_{r}$ over the radius value is defined by $M E_{r}=(1 / 1000)\left(\sum_{j=1}^{1000}\left|r-\hat{r}_{j}\right|\right)$, where $j$ indexes the trials and $\hat{r}_{j}$ is the radius estimation obtained at the $j$ th trial. The second criterion is the root mean square error $\mathrm{RMSE}_{r}$, defined by $\mathrm{RMSE}_{r}=\sqrt{(1 / 1000) \sum_{j=1}^{1000}\left(\hat{r}_{j}-r\right)^{2}}$.
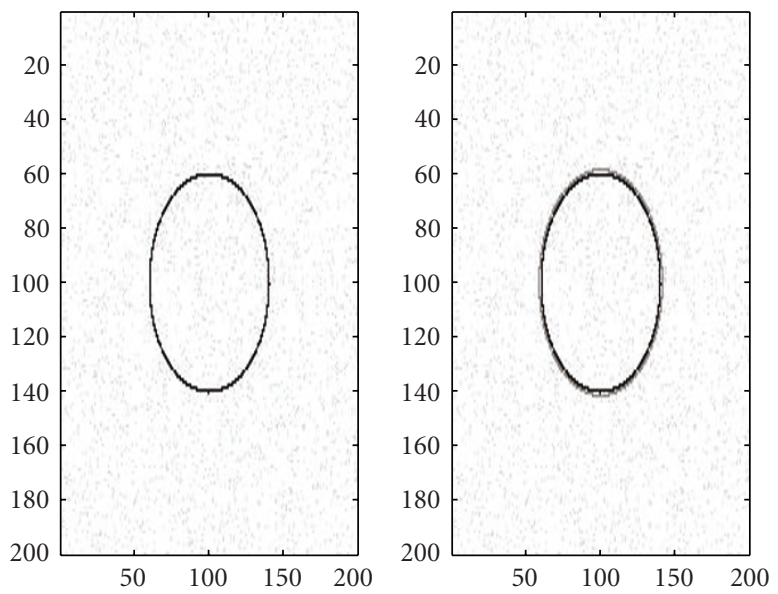

(a)
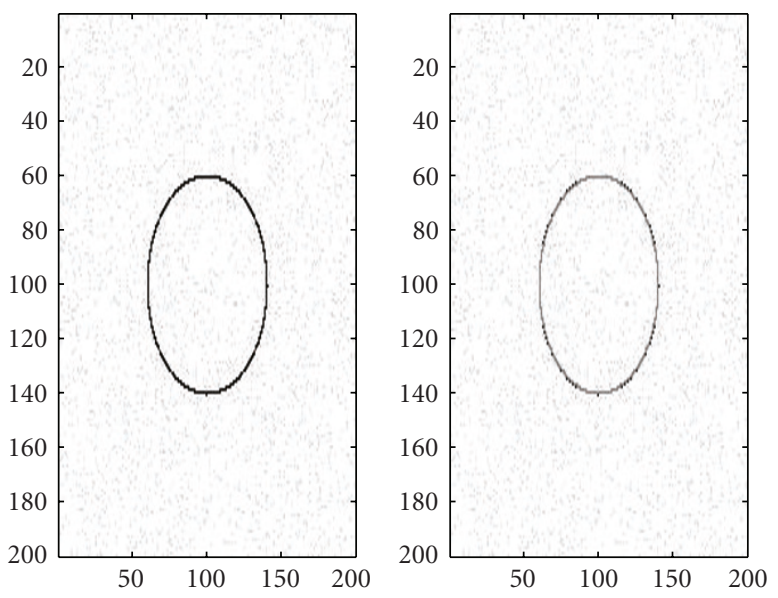

(b)

Figure 4: One circle: radius estimation by the proposed method and GHT: (a) processed image and result with our method, (b) processed image and result with GHT. 

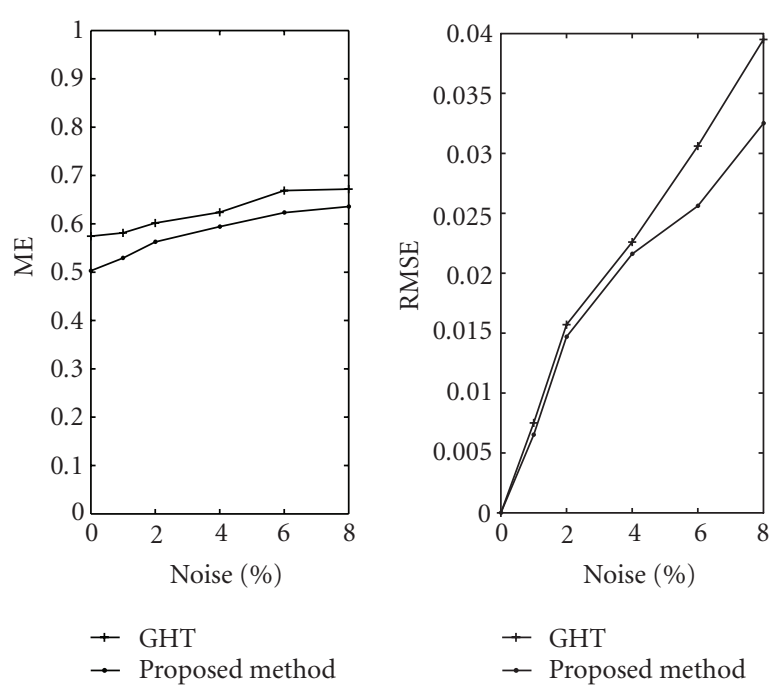

FIgURE 5: Mean value (pixels) and root mean square error (pixels) of the mean bias over the radius value, as a function of the percentage of noisy pixels.

The results presented in Figure 5 show that $M E_{r}$ values differ by less than 0.1 pixel and are always less than 1 pixel. $\mathrm{RMSE}_{r}$ values differ by less than 0.05 pixel. Therefore, statistical results obtained with both methods are very close, slightly better for the GHT, at the expense of a larger computational time.

\subsubsection{Circle fitting: real-world images}

Proposed methods can be applied to practical issues. We assume that all expected contours are centered on the middle of the image. We first give the result obtained by circle fitting (see Figure 6).

Figure 6 shows that the contour of each object presented is efficiently retrieved.

\subsubsection{Ellipse fitting and limitations}

An ellipse is no longer characterized by a constant radius, but by two axial parameters which are the largest and the smallest distances between the contour and its center. In the case where an ellipse is expected, the signal model proposed in (2) does not hold because one cannot define one constant frequency in the generated signal, as it was done in the case of a circle. However, when we perform the eigendecomposition of the covariance matrix of the recorded signal snapshots, we note that there exist two dominant eigenvalues. Therefore, the dimension of the signal subspace is fixed to two. Equation (6) leads to the two approximate values of the axial parameters of the ellipse. Then the proposed optimization method cancels the shift between the initialization ellipse and the expected contour. As a comparison, we expose the result obtained with the method proposed in [6] that leads to the ax- ial parameters of the ellipse through signal generation upon a linear antenna. Figure 7 shows the results obtained from an image containing a slightly distorted ellipse, with axial parameters 65 and 75 pixels. The estimated values provided by the method proposed in [6] are 65.6 and 65.6 pixels (see Figure $7(\mathrm{~b})$ ). The bias on one axial parameter can be due to the presence of a slight distortion. The estimation provided by the GHT, which aims at retrieving only one radius parameter, is 65.7 pixels (see Figure $7(\mathrm{c})$ ). The estimated values provided by our method are 67.0 and 78.6 pixels (see Figure $7(\mathrm{~d})$ ). The slight bias on these values can come from the distortion of the ellipse. This bias is lower than in the case of Figure 7(b); when the circular antenna is used, all sensors receive a nonzero signal component and then all components contribute in the estimation of the expected parameters. This permits to our circular antenna to cope more efficiently with slight distortions than when a linear antenna is used. When our method for retrieval of the distortions is run with 3000 iterations of gradient, with descent step parameter $\lambda=0.02$, the bias between initialization contour and expected contour is canceled (see Figures 7(e) and 7(f)). Then our method based on a circular antenna copes with the harsh case of a slightly distorted ellipse, for this we choose correctly the dimension of the signal subspace obtained from the generated signal. We consider now the case of an ellipse for which the ratio between axial parameters is far from unity. Figure 8 shows the results obtained from an image containing an ellipse with axial parameters 45 and 85 pixels. The estimated values provided by the method proposed in [6] are 47.3 and 88.6 pixels (see Figure $8(\mathrm{~b})$ ). The estimation provided by the GHT, which aims at retrieving only one radius parameter, is 45 pixels (see Figure $8(\mathrm{c})$ ). The estimated values provided by our method are 49.7 and 96.8 pixels (see Figure $8(\mathrm{~d})$ ). The bias on these values can come from a signal model which is not adequate. Then our method based on a circular antenna copes with the case of an ellipse whose axial parameters are close to each other but is limited as soon as the ratio between axial parameters is far from unity. This is due to the assumption of linear phase in the signal generated on the circular antenna (see (2)). In the next subsection we will focus on distorted circles.

\subsection{Distorted contours}

In this subsection we illustrate the performances of the optimization methods proposed for the estimation of the distortions between an initialization circle and the expected contour. We compare the abilities of our methods with the abilities of GVF. gradient algorithm, which is less robust but faster than DIRECT combined with spline interpolation, is employed for hand-made images. Descent step parameter is $\lambda=0.02$, and 3000 iterations are necessary.

\subsubsection{Illustration of the results obtained with gradient algorithm: hand-made images}

The result obtained in Figure 9 shows that even if there exists a bias between real and estimated values of the radius, 

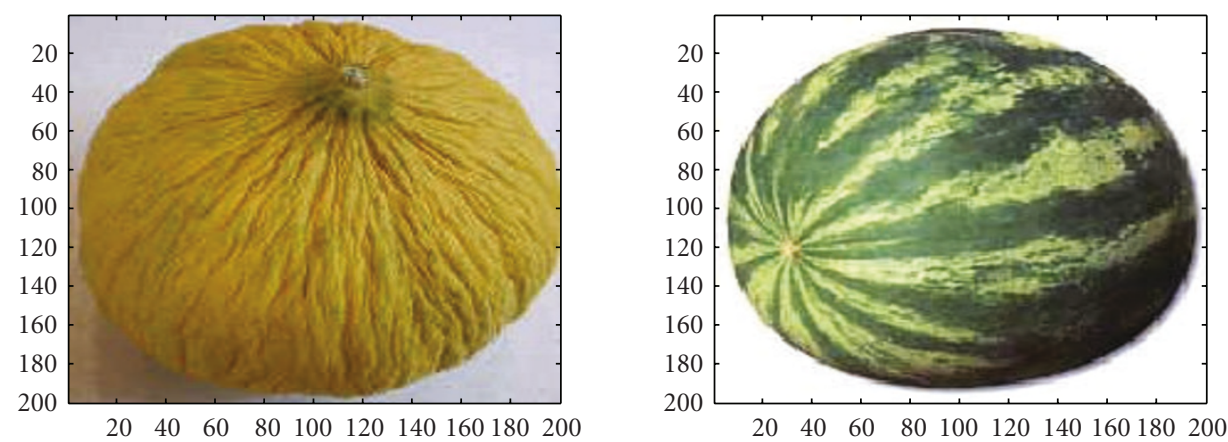

(a)
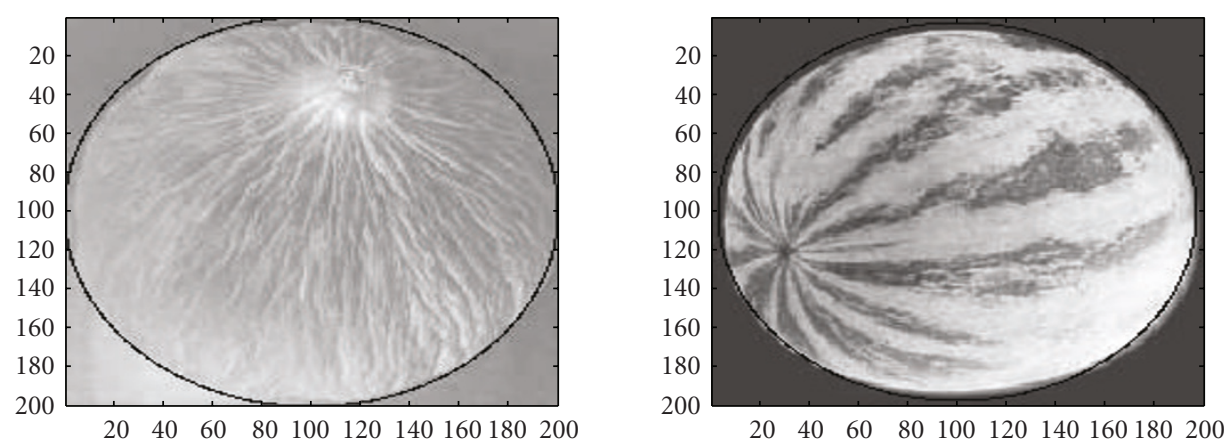

(b)

Figure 6: (a) Processed image, (b) result obtained with the proposed method for radius estimation. $M E_{\rho}=0.4$ and 0.6 pixel.

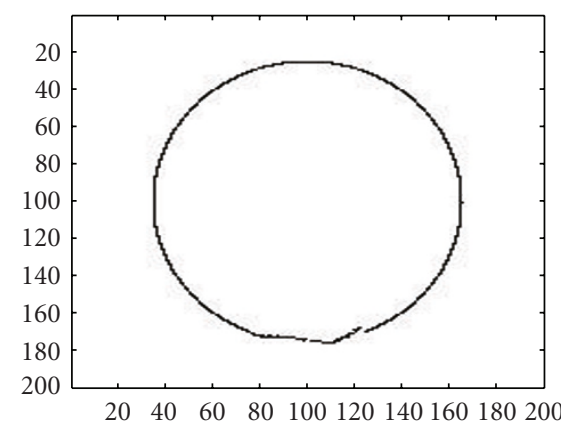

(a)

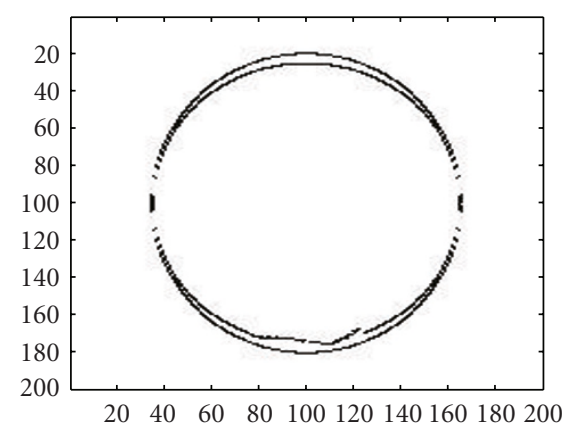

(d)

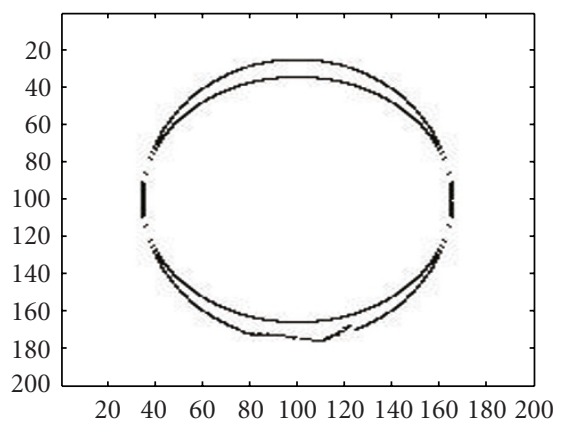

(b)

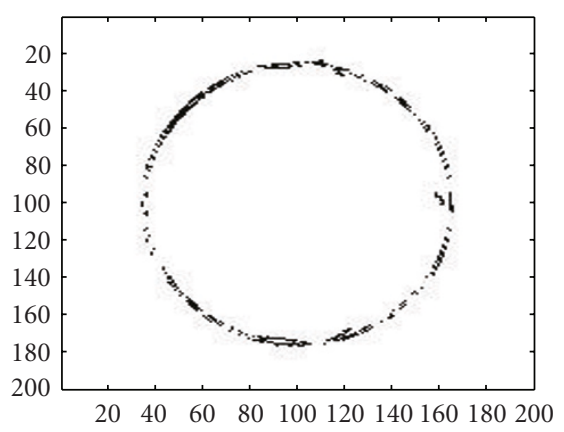

(e)

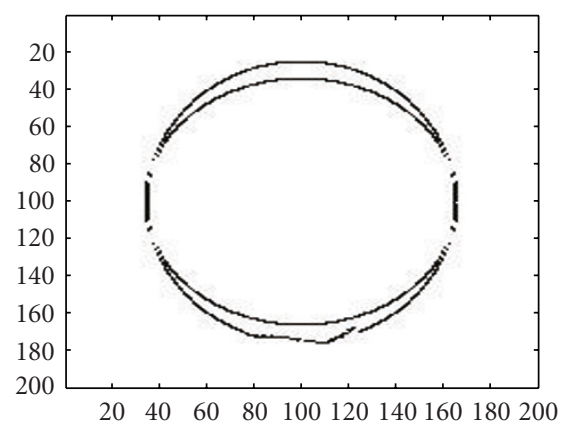

(c)

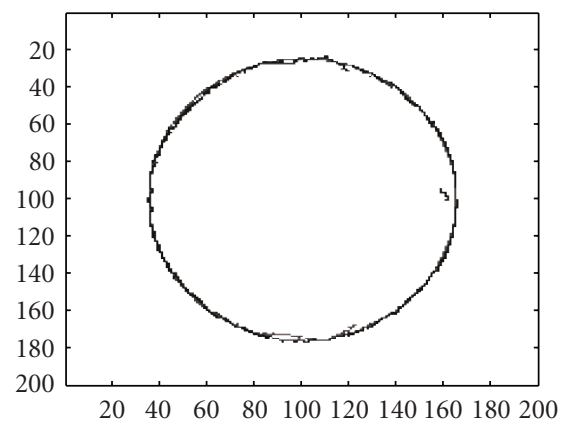

(f)

FIgURE 7: Ellipse fitting: (a) processed. (b) Difference processed and result by the existing method for ellipse retrieval [6]. (c) Difference processed and result by the GHT. (d) Difference processed and result obtained after applying the proposed method: $M E_{\rho}=2.8$ pixel. (e) Difference processed and result obtained after applying gradient method: $M E_{\rho}=0.7$ pixel. (f) Superposition processed and result obtained after applying gradient method. 


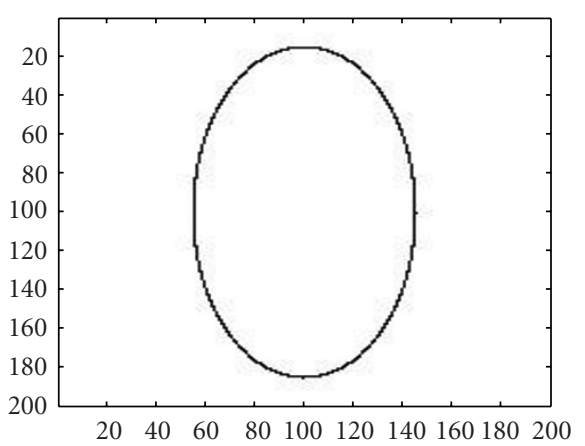

(a)

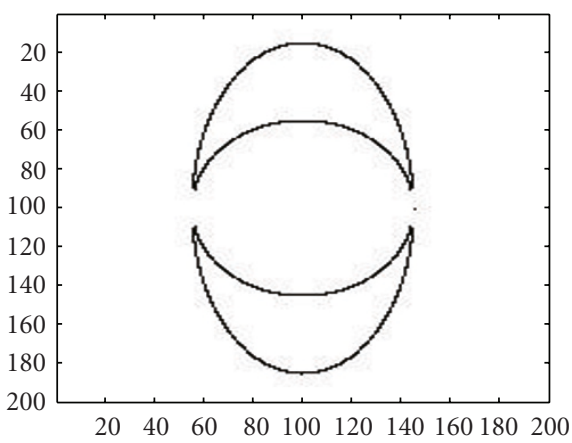

(c)

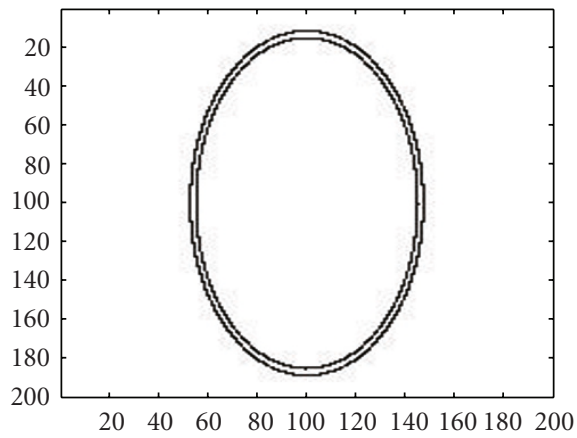

(b)

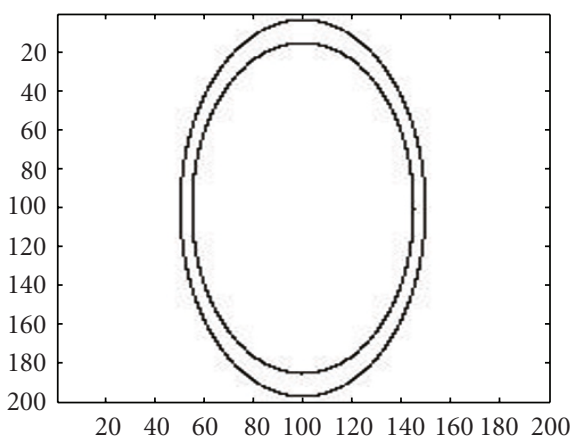

(d)

FIgURE 8: Ellipse fitting: (a) processed. (b) Difference processed and result by the existing method for ellipse retrieval [6]. (c) Difference processed and result by the GHT. (d) Difference processed and result obtained after applying the proposed method.

the optimization method finds the expected circle. The case of Figure 9 is not handled easily by GVF which has to be initialized close to the expected contour to converge in the same computational time as gradient.

We now consider the case of a noisy image. A leastsquares criterion gives an optimal result in the case of Gaussian noise. Then, as the proposed optimization methods are applied to minimize a least-squares criterion over the generated signal (see (9)), the result obtained should be optimal. We consider then a noisy image containing a slightly distorted circle. In order to impair our hand-made images, we add a Gaussian noise to a percentage $p$ of the pixels of the nonnoisy image. We adopt the same parameters as in Section 6.1.2. The image considered in Figure 10 is impaired by $p=20 \%$ of noisy pixels.

We also give the result obtained with GVF, from the same image, the same initialization contour and another noise realization with the same parameters. Indeed, there exists only one continuous contour to be retrieved and the initialization circle is close to the expected contour, which leads to a computational time which is the same as when the gradient optimization method is used. We choose the following parameters, that lead to a good result in terms of mean error and requires an acceptable computational time: parameter values are [8] $\alpha_{\mathrm{GVF}}=0.5$ (tension, rather elevated because of the presence of noise), $\beta_{\mathrm{GVF}}=0.01$ (rigidity), $\gamma_{\mathrm{GVF}}=1$ (regu- larization coefficient), $\kappa_{\mathrm{GVF}}=0.8$ (Gradient strength coefficient), $\mu_{\mathrm{GVF}}=0.15$ (regularization parameter in the GVF formulation), and 120 iterations are asked for the deformation.

Gradient method cancels the pixel shifts between the initialization circle and the expected circle. GVF method also cancels the pixel shifts. Computational (CPU) times which are needed for center estimation and radius estimation are, respectively, $3.8 \cdot 10^{-2}$ seconds and $7.8 \cdot 10^{-1}$ seconds. Signal generation lasts 0.14 seconds and fixed step gradient method lasts 1.9 seconds each time they are run. Thus 8.2 seconds are needed for the four quarters of image. Running GVF method lasts 9.1 seconds. We tested the variable step gradient method, which gives the same visual result and is ten times faster than the fixed step gradient method for this example. However, we will use fixed step gradient in the following. In this way, the performances of gradient and GVF in terms of mean error are evaluated for computational times that differ by only $10 \%$.

\subsubsection{Statistical results obtained with gradient algorithm applied to noisy hand-made images}

Fixed step gradient method and GVF [8] are applied to images containing a slightly-distorted circle. We adopt the same noise parameters as in Section 6.1.2, considering various 


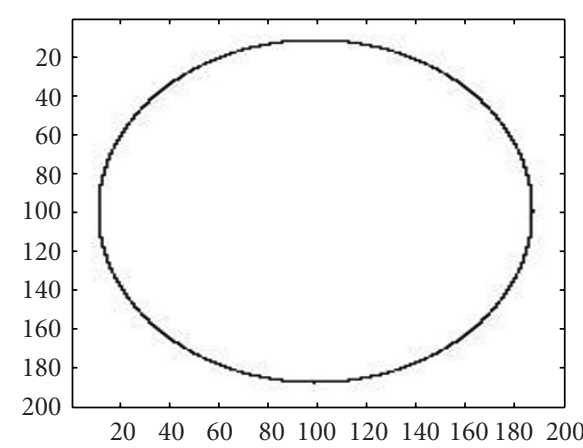

(a)

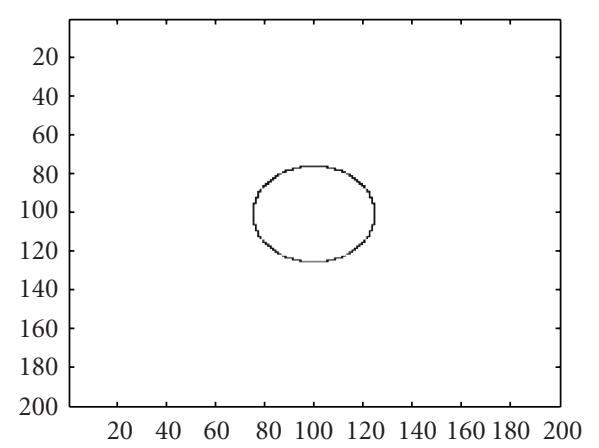

(b)

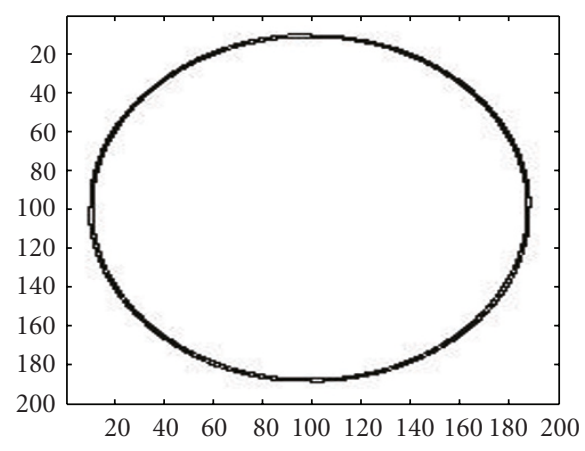

(c)

Figure 9: One circle: biased radius estimation, and application of gradient algorithm: (a) processed. (b) Initialization. (c) Superposition processed and result obtained after applying gradient method: $M E_{\rho}=0.15$ pixel.

noise percentage values. All these images are similar to the processed image of Figure 10. In order to evaluate only the performances of the proposed optimization method and GVF, both methods are initialized assuming the a priori knowledge of the center and the radius of the distorted circle. The parameters of signal generation and signal processing methods, for the proposed optimization method, are the same as in Section 6.1. Statistical results presented below are obtained with 15 images, each containing a different distorted circle. Noise parameters are the same as those employed for the study of radius estimation, propagation parameter is $\mu=5 \cdot 10^{-3} \cdot 1000$ iterations are necessary for gradient algorithm. Computational times are respectively 4.5 seconds for gradient algorithm on each quarter of image and 17 seconds for GVF method. So performances are compared for the same computational time. GVF method is run with the same parameter values as in Section 6.1. The first criterion that is employed to measure the accuracy of the results is the mean value of $M E_{\rho}$. Mean error $M E$ is defined by $M E=(1 / 1500) \sum_{i=1}^{15}\left(\sum_{j=1}^{100} M E_{\boldsymbol{\rho}_{i_{j}}}\right)$, where $j$ indexes the trials and $M E_{\boldsymbol{\rho}_{i_{j}}}$ is the mean error over all pixels of the contour obtained at the $j$ th trial for the $i$ th image. The second criterion is the root mean square error RMSE, defined by $\mathrm{RMSE}=\sqrt{(1 / 1500) \sum_{i=1}^{15} \sum_{j=1}^{100}\left(M E_{\boldsymbol{\rho}_{i_{j}}}\right)^{2}}$. The right image of Figure 11 shows that mean error values are less than one pixel for each noise percentage value and for both methods, thus, acceptable for many applications. The error values obtained with GVF method are between 11 and 27\% higher for the considered values of noise percentage. The low-root mean square error values show that both methods are robust to noise impairment. The left image of Figure 11 shows that the root mean square error values obtained with GVF are between 28 and $41 \%$ higher than the values obtained with the proposed method. The errors obtained with the proposed method are not due to the optimization method, which leads to a value zero for the criterion to be optimized. Errors come from the signal generation process: for noisy images the generated signal is corrupted and its phase exhibits unexpected fluctuations. The errors obtained with GVF come from a nonoptimal interplay between all parameters for all images.

\subsubsection{Distorted circle fitting: real-world images}

The parameters of signal generation and signal processing methods for radius estimation are the same as in Section 6.1. We assume that all expected contours are centered on the middle of the image. Real-world images are supposed to be harsher to process than hand-made images because of the presence of random noise and disruptions in the expected contours. Gradient algorithm would obligatorily focus on noise pixels in the disrupted sections of the expected contour. That is why we use the combination of the robust DIRECT method and spline interpolation which reduces the computational time of DIRECT and leads to a continuous result contour. Figure 12 gives the result obtained by gradient method and DIRECT combined with spline interpolation on the first real-world image, that concerns the practical issue of calibrating pies. DIRECT combined with spline is fast enough if a small number of nodes are chosen for the interpolation to be compared to the GVF method. Therefore, we also give the result obtained by GVF. Figure 12(a) gives the original color image. Figure 12(b) gives the initialization circle superimposed to the processed image. Figure 12(c) shows that gradient provides us with a contour which is not continuous and whose pixels go aside the pixels of the expected contour. When gradient method is employed, the mean error value $M E_{\rho}$ is 1.7 pixel. Parameters used to run gradient 


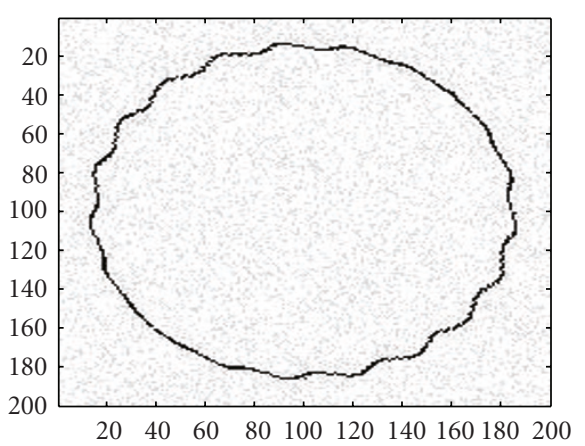

(a)

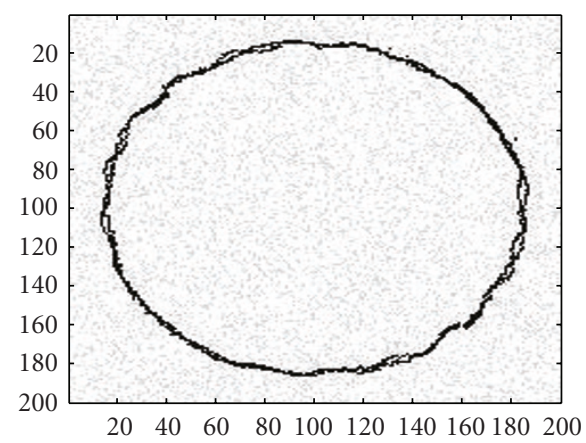

(c)

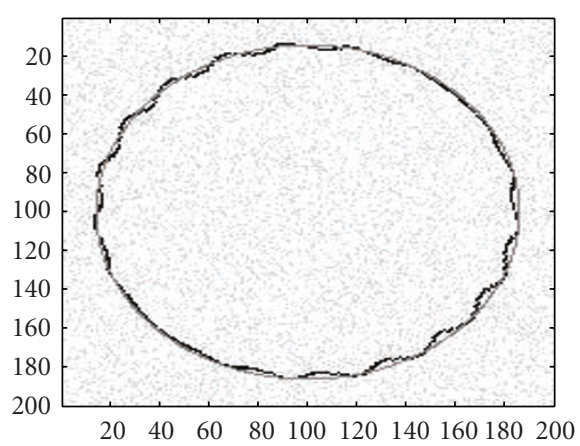

(b)

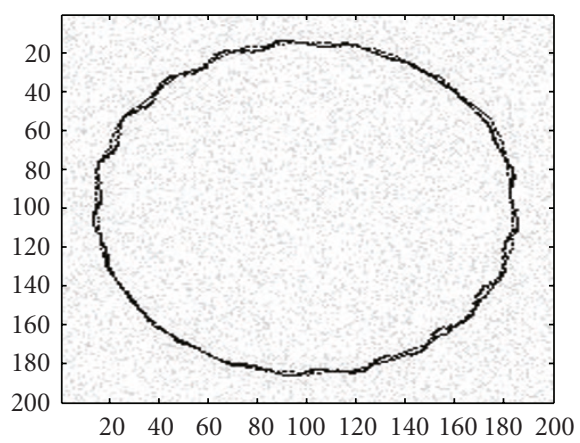

(d)

FIGURE 10: (a) Processed. (b) Initialization by the existing method for center estimation [6] and the proposed method for radius estimation. (c) Superposition processed and result obtained after applying gradient method: $M E_{\rho}=0.55$ pixel. (d) Superposition processed and result obtained after applying GVF method [8]: $M E_{\rho}=0.75$ pixel.
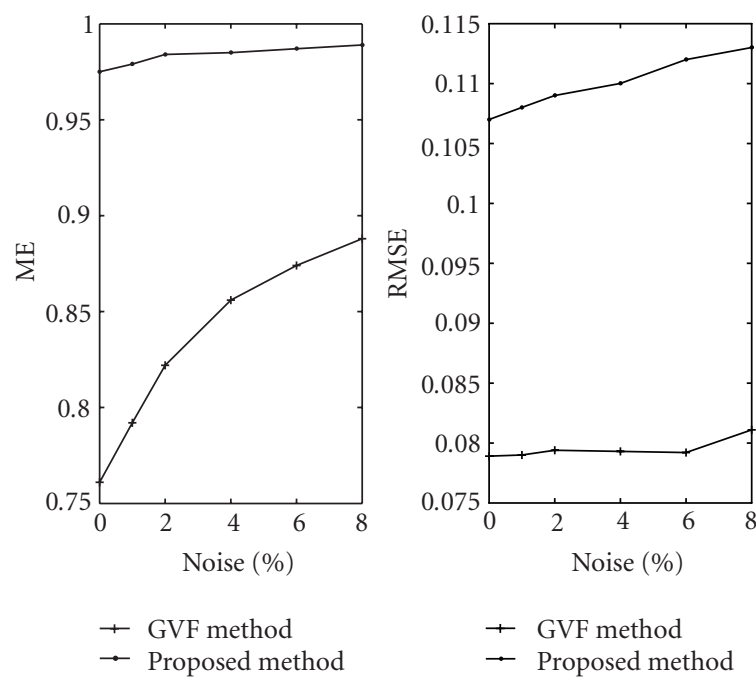

FIgURE 11: Mean value (pixels) and root mean square error (pixels), as a function of the percentage of noisy pixels, of the mean pixel bias value over the circle.

method are the same as the parameters used in Section 6.1. Figure 12 (d) gives the result obtained with GVF, run with the same parameters as in Section 6.2.2. Mean error value $M E_{\rho}$ is 1.9 pixel. With this method, the result obtained is continuous and exhibits low-curvature variations, but the numerous noise pixels prevent the active contour from converging exactly toward the expected contour.

Figure 12(e) gives the result obtained by DIRECT combined with spline interpolation. When this robust optimization method is used, the mean error value $M E_{\rho}$ is 1.6 pixel. Parameters used for running DIRECT and spline interpolation are the following: 6 interpolation nodes, and 5 iterations for DIRECT. Computational times are, respectively, 30 and 40 seconds for GVF and the proposed method.

\section{CONCLUSION}

We have shown in this paper how array processing and optimization methods can be applied to estimate distorted circular contours in images. In particular, we have shown the interest of the use of a circular antenna for the generation of linear phase signals when the processed image contains a circular contour. This facilitates the application of highresolution methods and optimization algorithms in the estimation of distorted circles in images. We proposed a method for the estimation of several possibly close radius values, and adapted an optimization strategy to the case of the retrieval 


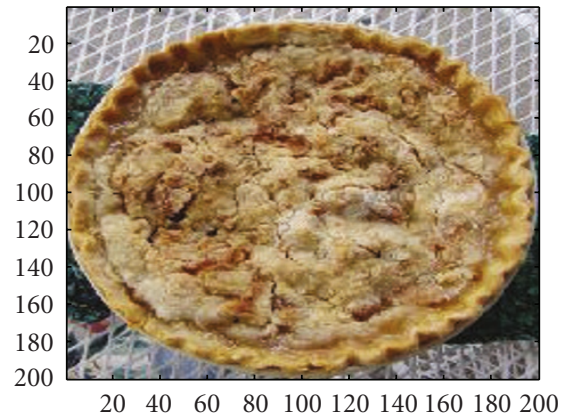

(a)

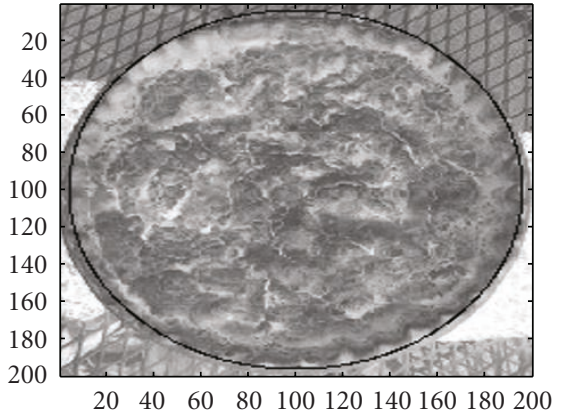

(b)

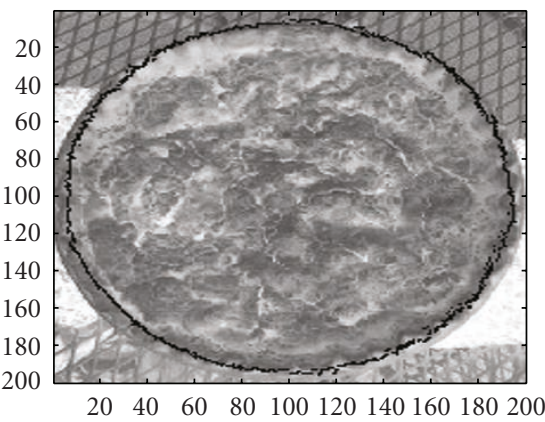

(c)

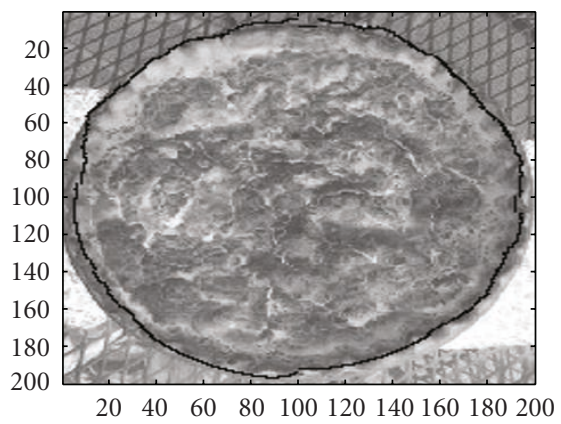

(d)

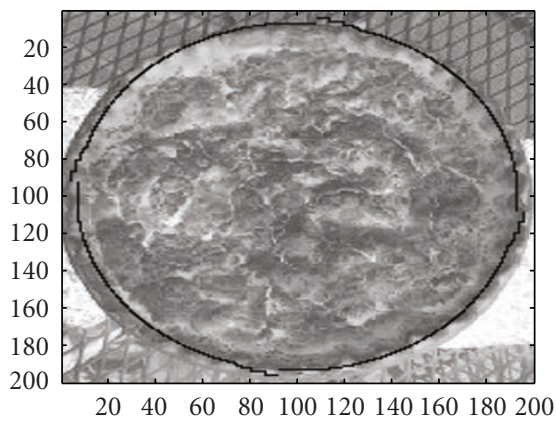

(e)

Figure 12: (a) Processed image. (b) Initialization. (c) Result obtained with gradient method. (d) Result obtained with GVF. (e) Result obtained with DIRECT combined with spline interpolation.

of distorted circles. The proposed method for radius estimation is faster than the generalized Hough transform and exhibits a good statistical behavior. This also holds for our optimization methods that we compared with GVF. We applied the robust optimization method based on DIRECT and spline interpolation to real-world images coming from practical issues. Real-world images were processed successfully, low pixel bias are obtained compared with the other considered methods. Further work could consist in estimating the parameters of multiple circles with different radius and also different center.

\section{ACKNOWLEDGMENT}

The authors would like to thank the anonymous reviewers who contributed to the quality of this paper by providing helpful suggestions.

\section{REFERENCES}

[1] J. F. Crawford, "A noniterative method for fitting circular arcs to measured points," Nuclear Instruments and Methods in Physics Research, vol. 211, no. 2, pp. 223-225, 1983.

[2] V. Karimäki, "Effective circle fitting for particle trajectories," Nuclear Instruments and Methods in Physics Research Section A, vol. 305, no. 1, pp. 187-191, 1991.
[3] G. Coath and P. Musumeci, "Adaptive arc fitting for ball detection in robocup," in Proceedings of APRS Workshop on Digital Image Computing (WDIC '03), pp. 63-68, Brisbane, Australia, February 2003.

[4] D. H. Ballard, "Generalizing the Hough transform to detect arbitrary shapes," Pattern Recognition, vol. 13, no. 2, pp. 111$122,1981$.

[5] C. L. Tisse, L. Martin, L. Torres, and M. Robert, "Person identification technique using human iris recognition," in International Conference on Vision Interface (VI '02), pp. 294-299, Calgary, Canada, May 2002.

[6] H. K. Aghajan, "Subspace techniques for image understanding and computer vision," Ph.D. dissertation, Stanford University, Stanford, Calif, USA, 1995.

[7] H. K. Aghajan and T. Kailath, "Sensor array processing techniques for super resolution multi-line-fitting and straight edge detection," IEEE Transactions on Image Processing, vol. 2, no. 4, pp. 454-465, 1993.

[8] C. Xu and J. L. Prince, "Gradient vector flow: a new external force for snakes," in Proceedings of the IEEE Computer Society Conference on Computer Vision and Pattern Recognition, pp. 66-71, San Juan, Puerto Rico, USA, June 1997.

[9] X. Xie and M. Mirmehdi, "RAGS: region-aided geometric snake," IEEE Transactions on Image Processing, vol. 13, no. 5, pp. 640-652, 2004.

[10] S. Bourennane and J. Marot, "Contour estimation by array processing methods," EURASIP Journal on Applied Signal Processing, vol. 2006, Article ID 95634, 15 pages, 2006. 
[11] D. R. Jones, C. D. Perttunen, and B. E. Stuckman, "Lipschitzian optimization without the Lipschitz constant," Journal of Optimization Theory and Applications, vol. 79, no. 1, pp. 157-181, 1993.

[12] S. Bourennane and J. Marot, "Optimization and interpolation for distorted contour estimation," in Proceedings of IEEE International Conference on Acoustics, Speech and Signal Processing (ICASSP '06), vol. 2, pp. 717-720, Toulouse, France, May 2006.

[13] H. K. Aghajan and T. Kailath, "SLIDE: subspace-based line detection," IEEE Transactions on Pattern Analysis and Machine Intelligence, vol. 16, no. 11, pp. 1057-1073, 1994.

[14] R. Roy and T. Kailath, "ESPRIT-estimation of signal parameters via rotational invariance techniques," IEEE Transactions on Acoustics, Speech, and Signal Processing, vol. 37, no. 7, pp. 984-995, 1989.

[15] H. K. Aghajan, B. H. Khalaj, and T. Kailath, "Estimation of multiple 2-D uniform motions by SLIDE: subspace-based line detection," IEEE Transactions on Image Processing, vol. 8, no. 4, pp. 517-526, 1999.

[16] J. Sheinvald and N. Kiryati, "On the magic of SLIDE," Machine Vision and Applications, vol. 9, no. 5-6, pp. 251-261, 1997.

Julien Marot received the Physics engineering degree from ENSP Marseille, France, in 2003 and the Image Processing DEA in 2004. Since December 2004, he works as Ph.D. student in the multidimensional signal processing group (GSM), Fresnel Institute (CNRS UMR-6133). His research interests include applied image processing and signal processing.

Salah Bourennane received his Ph.D. degree from Institut National Polytechnique de Grenoble, France, in signal processing. Currently, he is Full Professor at the Ecole Centrale de Marseille, France. His research interests are in statistical signal processing, array processing, image processing, multidimensional signal processing, and performances analysis.
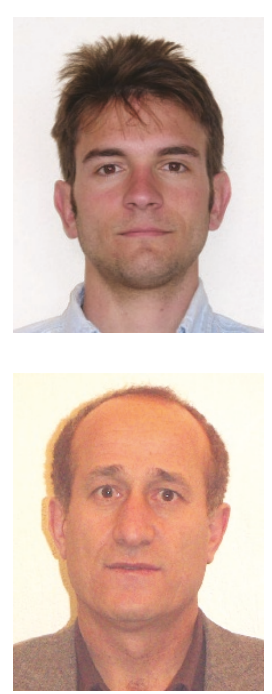